\title{
Device Markings/Labeling Problem
}

National Cancer Institute

\section{Source}

National Cancer Institute. Device Markings/Labeling Problem. NCI Thesaurus. Code C62976.

Problem associated with the written, printed or graphic material accompanying or affixed to the device or any of its packaging. This includes verbal instructions relating to identification, technical description, and usage provided by a medical device manufacturers. Problems can include but are not limited to this material being unclear, missing, worn out, incorrect or inaccurate. 